\title{
Winds of change in medical education in Singapore: what does the future hold?
}

\author{
Lionel Hon Wai $\underline{L u m}^{1}$, MBBS, MRCP, Kian-Keong $\underline{P o h^{2}}$, FRCP, FACC, Paul Anantharajah Tambyah ${ }^{1}$, MD
}

T he global landscape of medical education has evolved significantly over the past decade. While in the past, doctors largely learned by apprenticeship and medical education seemed to be governed by the 'see one, do one, teach one' principle, this is no longer considered acceptable in the modern technological era. Medical education in Singapore has developed in a different dimension in relation to the rest of the world. ${ }^{(1,2)}$

In the past decade, most undergraduate medical schools placed more emphasis on integrating basic sciences into clinical medicine, and vice versa. ${ }^{(1)}$ Simulation and case-based learning are now well established in the preclinical years. Other important facets of professional development such as medical ethics, cultivating 'soft' skills such as communications, and understanding healthcare policies and finances have also been introduced in the undergraduate curriculum ${ }^{(1)}$ in Singapore and globally. In the last decade, Yong Loo Lin School of Medicine, National University of Singapore (NUS Medicine) was joined by two other medical schools, Duke-NUS Medical School and Lee Kong Chian School of Medicine, creating an element of friendly competition in Singapore medical education. NUS Medicine has implemented entrustable professional activities, or EPAs, that detail the knowledge, skills and attributes of its desired graduate. ${ }^{(3)}$ The final MBBS (Bachelor of Medicine, Bachelor of Surgery) assessment has also taken a more comprehensive approach, introducing scenario-based modified essay questions on top of the traditional clinical short and long cases, and written examinations. The student internship programme, which prepares students for housemanship, is now a well-established part of the medical curriculum at the school. At the same time, the two newer medical schools have advanced even more innovative educational approaches, including team-based learning and mandatory interprofessional education in clinical rotations. ${ }^{(4,5)}$

While these changes are in line with global trends and best practices, a monumental change in postgraduate medical education in Singapore also took place in the last decade. In the quest for better-quality specialty training as well as to meet the rising need for specialists in the late 2000s, Singapore shifted from the basic and advanced specialty training system to the United States (US) residency system under the purview of the Accreditation Council for Graduate Medical EducationInternational. ${ }^{(1,2,6)}$ Most Singaporean specialists had been trained in a looser competency-based system where trainees rotated through different departments until they were able to demonstrate their clinical competency in rigorous clinical examinations. This gave way to an American system that had a more structured programme with clearly defined core competencies, greater documentation of supervision from the designated faculty, more rigorous formative assessments and feedback sessions, stipulated protected time for learning, and fixed time periods for basic and advanced training. Overall, this demanded greater accountability as well as documentation from both trainees and educators, while producing significant numbers of trainees who had completed the programme requirements.

As we attempt to understand the impact of the new training system, we realise that a 'utopia' in medical education is very difficult to achieve. The adoption of the US residency system has had an impact that extends into the medical school years. Until recently, the majority of the graduating medical students would have decided on their choice of postgraduate residency programme during their final year of medical school, which has been described as being almost akin to a blind date, given the nature of medical school education in Singapore. In this issue of the Singapore Medical Journal, $\mathrm{Ng}$ et $\mathrm{al}^{(7)}$ and Fong et $\mathrm{a}^{\left({ }^{(8)}\right.}$ explore the various reasons that students give for choosing their postgraduate training programme. These include their limited undergraduate experience with the institution and their financial background. While these may be significant factors influencing the choice of residency and institution in any setting, enrolment into a residency should be primarily based on a candidate's aptitude and interests. The best fit can probably only be confirmed after a period of time and through exposure to different subspecialties including generalist rotations and family practice. This has been recognised, and applications for residency can now only be initiated after a year of rotating housemanship postings. The role of mentors and seniors as career counsellors, which many of us recall from our past, is another area that may need to be developed.

Beyond the choice of postgraduate training programme, there is growing recognition that it is vital to strike a balance between clinical services and protected learning time. While work hours are restricted in the current US and US-style residency system, 'hands-on' time (particularly in the surgical disciplines) may be reduced. ${ }^{(9,10)}$ The exponential growth of electronic paperwork mandated by new electronic medical records systems worldwide and the concurrent surge in the number of specialist trainees locally have further decreased clinical opportunities for trainees. In an attempt to ensure the welfare of residents as well as patient safety in this environment, several institutions have adopted a night float on-call system. The impact of a night float compared 
with the traditional overnight on-call system has been studied by Low et $\mathrm{al}^{(11)}$ in relation to the sleep quality and fatigue rates of residents, with some surprising results. It is clear that we will have to work out a system that protects both patients and trainees from fatigued residents while ensuring optimal clinical training in a complex environment. It will not be easy, but studies like these are important and necessary steps.

Lastly, more resources have been channelled into equipping educators. The annual Asia Pacific Medical Education Conference and the Master of Health Professions Education Singapore and Centre for Medical Education programmes are among the few available local platforms. Increasingly, we are seeing more innovative methods of teaching. Flipped classrooms, augmented reality and gamification are among the most prominent - but whether these translate into better teaching outcomes requires further in-depth studies. ${ }^{(1)}$

It has been argued that beyond pedagogical sophistication, the individual qualities of the students and trainees are more important. It is thus reassuring that Lean et $\mathrm{a}^{\left({ }^{12)}\right.}$ have suggested that NUS Year 4 medical students are more likely to have the desired traits of a doctor compared with students from other faculties. The challenge is to nurture and maximise the potential of these future doctors, and to sustain their passion in medicine through the long and rigorous medical training journey.

The original research articles in this issue provide small but critical insights into the impact of the many changes in medical education in Singapore. There are certainly many more initiatives in place in the different medical schools that need to be critically evaluated. Singapore has just only stepped through the looking glass into the complex world of medical education for the 21 st century. The Singapore brand name is strong in many fields, especially education, and perhaps now is the time to develop a robust and holistic Singaporean postgraduate medical education system that incorporates some of the best elements from across the world with local evidence-based innovations. As we do so (and it seems inevitable that we will), it is vital that we constantly evaluate the changes made using the vast quantities of data collected $^{(13)}$ so that we can respond dynamically to the rapidly evolving global medical education scene.

\section{REFERENCES}

1. Samarasekera DD, Ooi S, Yeo SP, Hooi SC. Medical education in Singapore. Med Teach 2015; 37:707-13.

2. Mahendran R, Broekman B, Wong JCM, Lai YM, Kua EH. The educational environment: comparisons of the British and American postgraduate psychiatry training programmes in an Asian setting. Med Teach 2013; 35:959-61.

3. Goh DLM, Samarasekera DD, Jacobs JL. Implementing entrustable professional activities at Yong Loo Lin School of Medicine Singapore undergraduate medical education program. South East Asian J Med Educ 2015; 9:5-14.

4. Kamei RK, Cook S, Puthucheary J, Starmer CF. 21st century learning in medicine: traditional teaching versus team-based learning. Med Sci Educ 2012; 22:57-64.

5. Rajalingam P, Rotgans JI, Zary N, et al. Implementation of team-based learning on a large scale: three factors to keep in mind. Med Teach 2018; 40:582-8.

6. Ministry of Health, Singapore. Graduate Medical Education in Singapore. Available at: http://www.healthprofessionals.gov.sg/sab/specialist-training/ graduate-medical-education-in-singapore. Accessed September 14, 2018.

7. $\mathrm{Ng} \mathrm{CL}$, Liu XD, Murali Govind R, et al. Factors affecting choice of sponsoring institution for residency among medical students in Singapore. Singapore Med J 2018; 59:642-6.

8. Fong JM, Tan YT, Sayampanathan AA, et al. Financial background and student debt impact on postgraduate residency choices of medical students in Singapore. Singapore Med J 2018; 59:647-51.

9. Sim KS, Teo JY, Cheow PC. Changes in operative experience after the introduction of residency: an objective comparison of surgical and endoscopic volumes. Singapore Med J 2018; 59:500-4.

10. Teo JY, Lee SY, Chua AJ, Wong WK. Will the local ACGME-trained surgeon be adequately prepared? An estimate of the impact of duty hour restrictions on operative experience. Ann Acad Med Singapore 2013; 42:203-6.

11. Low JM, Tan MY, See KC, Aw MM. Sleep, activity and fatigue reported by postgraduate year 1 residents: a prospective cohort study comparing the effects of night-float versus traditional overnight on-call. Singapore Med J 2018; 59:652-5.

12. Lean LL, Hong RYS, Ti LK. How the personalities of medical students at the National University of Singapore differ from the local non-medical undergraduate population: a cross-sectional study. Singapore Med J 2018; 59:656-9.

13. Koh GCH, Lee JNE, Agrawal N, et al. The Impact of Education Reform: An Asian Medical School's Experience. Ann Acad Med Singapore 2016; 45:198-204. 